\title{
Comparison of Anterior Chamber Parameters between Normal and Keratoconus Eyes using Scheimpflug Photography
}

\author{
${ }^{1}$ Nagla Hassan Ali, ${ }^{2}$ Mohammed Othman AbdEIKhalek, ${ }^{3}$ Hanan Elghoneimy
}

\begin{abstract}
Aim: To evaluate anterior chamber indices including anterior chamber depth (ACD) and anterior chamber volume (ACV) with the progression of keratoconus using a rotating Scheimpflug camera to determine the sensitivity and specificity of these parameters in discriminating keratoconus from normal eyes.

Materials and methods: After full ophthalmological examination in the Memorial Institute of Ophthalmic Research, the patients are divided into two groups: Group I consisting of 50 eyes of normal control. Group II consists of 50 eyes of moderate keratoconus. All the subjects were evaluated with a Pentacam Scheimpflug camera (Oculus, Wetzlar, Germany). Keratoconic eyes were evaluated by vertical asymmetry $(\mathrm{VI})$, keratoconus index $(\mathrm{KI})$, thinnest corneal thickness (TCT) with front elevation at thinnest location (F.Ele.Th) and back elevation at thinnest location (B.Ele.Th) and then divided into three groups according to mean keratometer $(K)$ readings: Mild ( $\mathrm{K}=$ less than 47.0 D), moderate ( $\mathrm{K}=47.0$ to $52.0 \mathrm{D})$, and severe ( $K=52.0 \mathrm{D}$ or higher), and $A C D$ and $A C V$ were obtained after exclusion of mild and severe cases.
\end{abstract}

Results: With the progression of the disease, there was statistically significant differences in ACD and ACV measurements between the normal and moderate keratoconus eyes with progressive increase in the ACD and ACV with disease progression.

Conclusion: Anterior chamber parameters including depth and volume showed significant increase with moderate keratoconus than control.

Keywords: Anterior, Photography, Scheimpflug.

How to cite this article: Ali $\mathrm{NH}$, AbdEIKhalek MO, El Ghonemy HM. Comparison of Anterior Chamber Parameters between Normal and Keratoconus Eyes using Scheimpflug Photography. Int J Kerat Ect Cor Dis 2016;5(3):105-108.

\section{Source of support: Nil}

Conflict of interest: None

\section{INTRODUCTION}

Oculus Pentacam combines a rotating Scheimpflug camera with a static camera to acquire multiple photographs

\footnotetext{
${ }^{1,2}$ Lecturer, ${ }^{3}$ Assistant Consultant

1,3Memorial Institute of Ophthalmological Research, Giza, Egypt

${ }^{2}$ Faculty of Medicine Beni Suef University, Beni Suef, Egypt
}

Corresponding Author: Nagla Hassan Ali, Lecturer, Memorial Institute of Ophthalmological Research, Giza, Egypt, Phone: +00201005293288, e-mail: naglahas@yahoo.com of the anterior eye segment. The Scheimpflug camera rotates along with a monochromatic slit-light source around the optical axis to obtain the slit images. This rotating system performs a corneal scan from zero to $180^{\circ}$ and each one of the photographs is an image of the cornea at a specific angle.

Scheimpflug imaging systems are based on a principle that allows documentation of an object not parallel to the lens and image planes of a camera. It works with maximal possible depth of focus and minimal image distortion. These systems can image and provide meaningful information starting from the anterior corneal surface. ${ }^{1}$

The photographs are used in the reconstruction of the anterior and posterior corneal topographies from height data. Analyses of corneal pachymetry, topography, densitometry, and the complete anterior chamber including depth and volume are also provided by the Pentacam. The Pentacam is a relatively new instrument with a rotating Scheimpflug camera that can take up to 50 Scheimpflug images of the anterior eye in less than 2 seconds. It is designed to measure five aspects of the anterior eye, namely front-surface topography of the cornea (FST), back-surface topography of the cornea (BST), thinnest corneal thickness (TCT), anterior chamber depth (ACD) and anterior chamber volume (ACV), and lenticular density. ${ }^{2}$

Keratoconus is an ectatic, noninflammatory disorder in which corneal thinning and protrusion cause the cornea to assume a conical shape. It is most commonly bilateral and asymmetric, with no gender or race predilection, and begins typically at puberty. ${ }^{3}$ Detection of initial (mild) and subclinical (forme fruste) keratoconus is of paramount importance in the preoperative evaluation of refractive surgery candidates to avoid complications, such as post-LASIK ectasia.

Studying anterior chamber parameters including depth and volume in patients with moderate keratoconus by using Pentacam showed that the instrument was capable of measuring test surfaces with high level of accuracy. Anterior corneal keratometry, posterior corneal keratometry and pachymetry (at the apex and thinnest point), and topography with $\mathrm{ACD}$ and $\mathrm{ACV}$, all are important aspects for diagnosis of keratoconus, 
however, it was also reported that early changes in eyes with $\mathrm{KC}$ are also present earlier at the posterior corneal surface. ${ }^{4}$

\section{MATERIALS AND METHODS}

This study analyzed 100 eyes of adult persons of age 20 to 35 years at the Memorial Institute of Ophthalmic Research and were divided into two groups. Group I consists of 50 eyes of normal control. Group II is of 50 eyes diagnosed as moderate keratoconus. The subjects were informed about the aims of the study and they gave verbal informed consent.

The control group consisted of normal healthy subjects who were staffs and visitors of the hospital volunteered to participate in the study. They were given a brief explanation about the procedures and consent was obtained from all participants.

All participants underwent a visual examination, including retinoscopy (without application of cycloplegia) followed by subjective refraction, slit-lamp biomicroscopy, and Pentacam tomographic evaluation. The inclusion criteria included normal healthy participants free from systemic and ocular diseases with refractive errors of less than $\pm 4.00 \mathrm{D}$ sphere and astigmatism less than 2.00 D. Best-corrected visual acuity was at least $6 / 9$ or better in each eye. Participants with a history of contact lens wear within the last 1 month, previous ocular surgery, ocular trauma, scarring, or ocular pathologies other than keratoconus were excluded from this study.

The criterion for diagnosis of the keratoconus was a scissoring reflex on retinoscopy, central or paracentral steepening of the cornea in topographic examination, or at least 1 of the subsequent biomicroscopic signs: Conical protrusion, stromal thinning, Vogt striae, Fleischer's ring, or scarring. Keratoconus subjects were classified into mild, moderate, and severe according to the mean keratometry readings driven from Pentacam and keratoconus index (KI) (mild: K $\leq 47.0$ D, moderate: $47.0<\mathrm{K}<52.0 \mathrm{D}$, and severe: $\mathrm{K} \geq 52.0 \mathrm{D})$. Only moderate keratoconus cases were included.

Pentacam high-resolution tomographer (Oculus, Wetzlar, Germany) was used for anterior segment imaging of the eyes. For this measurement, the examination room was set to dim-light condition. After adjusting the subject's chin and forehead on the chinrest and forehead rest, the subject was instructed to focus on the black spot in the middle of the blue fixation beam with both eyes open. The examiner then used the joystick to focus the image and obtain the correct alignment. Once this was obtained, the instrument captured 25 Scheimpflug images automatically within 2 seconds. This instrument is based on a $180^{\circ}$ rotating Scheimpflug camera that provides three-dimensional (3D) image of the anterior segment and calculates the parameters. The instrument takes about 20 seconds to process the images after which the next set of images was acquired. The subject removed his head from the rests during the time it took the instrument to process the images.

When the processing was complete the subject replaced his head on the rests, the instrument was realigned, and when the alignment was complete the next 25 Scheimpflug images were captured. Keratometric data was analyzed using software developed by Harris et $\mathrm{al}^{5}$ with modifications by Rubin. The Pentacam data was analyzed using commercially available statistical software and moderate keratoconus were diagnosed according to $\mathrm{K}$ reading flat and steep (47-52 D), positive anterior and posterior corneal elevation maps progression index average and positive keratoconus indices, pachymetry at pupil center and thinnest location, ACD and ACV.

Anterior chamber depth was defined as the distance from the corneal endothelium to the anterior surface of the lens capsule. The ACV is calculated from endothelium down to iris and lens over a $12 \mathrm{~mm}$ diameter centered on the anterior corneal apex.

Finally, a comparative study between the mean of $\mathrm{ACD}$ and ACV in moderate stages of keratoconus and control (Graph 1) was obtained and a statistical analysis was done by t-test with $\mathrm{p}$-value significant when it is below 0.05 .

\section{Exclusion Criteria}

The exclusion criteria included mild and severe degrees of keratoconus, any other corneal surface ectasia, and other corneal abnormalities like glaucoma, corneal scarring, severe eye dryness, current corneal infections, history of ocular surgery, and patients with history of chronic systemic diseases.

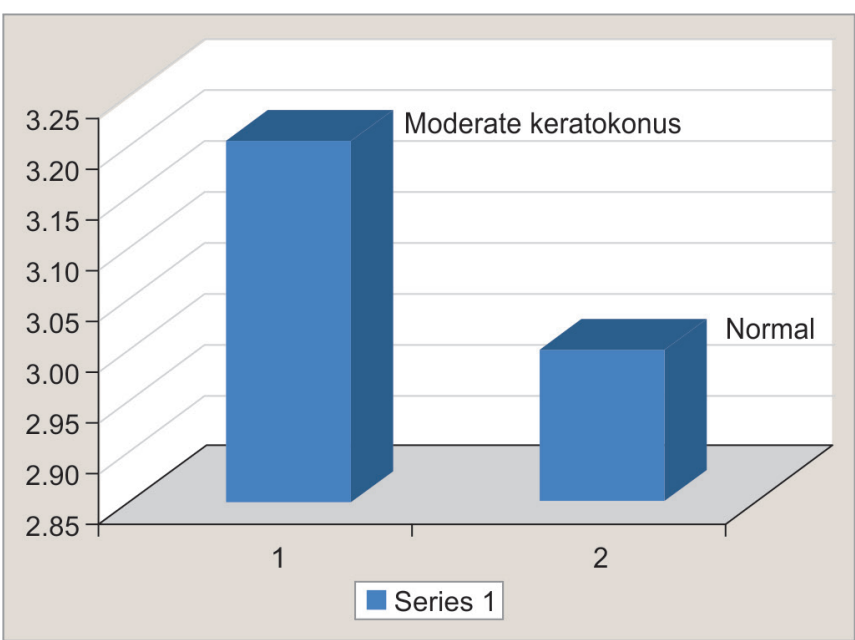

Graph 1: Example of a case of moderate keratoconus stage 2 with positive $\mathrm{K}$ indices 


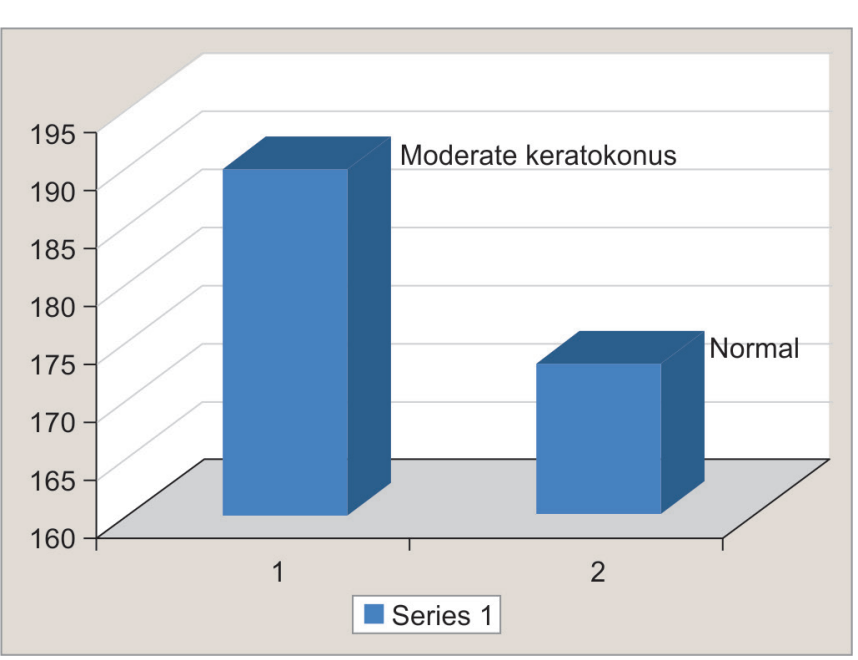

Graph 2: Anterior chamber depth in normal and moderate keratoconus. Normal subjects mean $( \pm 3.00 \mathrm{~mm})$ and $\mathrm{SD}( \pm 0.512 \mathrm{~mm})$ and with significantly higher mean ACD in moderate keratoconus $( \pm 3.204 \mathrm{~mm})$ with SD $( \pm 0.322 \mathrm{~mm})$

\section{RESULTS}

The study included group I of 50 eyes of 25 normal subjects, 12 males and 13 females in the age range 20 to 35 years, and group II included 50 eyes of 35 patients, 17 males and 18 females in the age range 20 to 35 years. There was no statistical difference between the two groups regarding age and gender.

By grading keratoconus according to dioptric power and according to Pentacam keratoconus map display, the results of the study were analyzed for patients with mean $\mathrm{K}$ reading \pm 49.385 and $\mathrm{SD} \pm 222$ and showed that the mean ACD of 50 eyes of normal subjects $(3.00 \pm$ $0.5124 \mathrm{~mm}$ ) was significantly less than in 50 eyes of moderate keratoconus patients with significantly higher mean $\operatorname{ACD}(3.204 \pm 0.322 \mathrm{~mm})$ than normal eye with $\mathrm{p}$-value 0.01 (Graph 2). The mean ACV of 50 eyes of normal subjects $\left(173 \pm 54.92 \mathrm{~mm}^{3}\right)$ was significantly less than in 50 eyes of moderate keratoconus patients with significantly higher mean ACD $\left(190 \pm 32.21 \mathrm{~mm}^{3}\right)$ than normal with p-value of 0.024 (Graph 3).

\section{CONCLUSION}

In the present study, the value of central ACD in normal group is lower than those of Edmonds et $\mathrm{al}^{6}$ that used Scheimpflug photography $(3.00 \pm 0.4124$ vs $3.18 \pm$ $0.32 \mathrm{~mm}$ ) and were in agreement with Abolbashari et al. ${ }^{7}$ The central ACD in keratoconus and its changes with keratoconus progression is in agreement with the results of Emre et $\mathrm{l}^{8}$ study who using Pentacam found progressively longer ACD values at center in mild, moderate, and severe keratoconus subjects with the highest values in the last group. According to their study, the mean $\mathrm{ACD}$ at the center was $3.2 \pm 0.3,3.3 \pm 0.3$, and $3.7 \pm 0.4 \mathrm{~mm}$

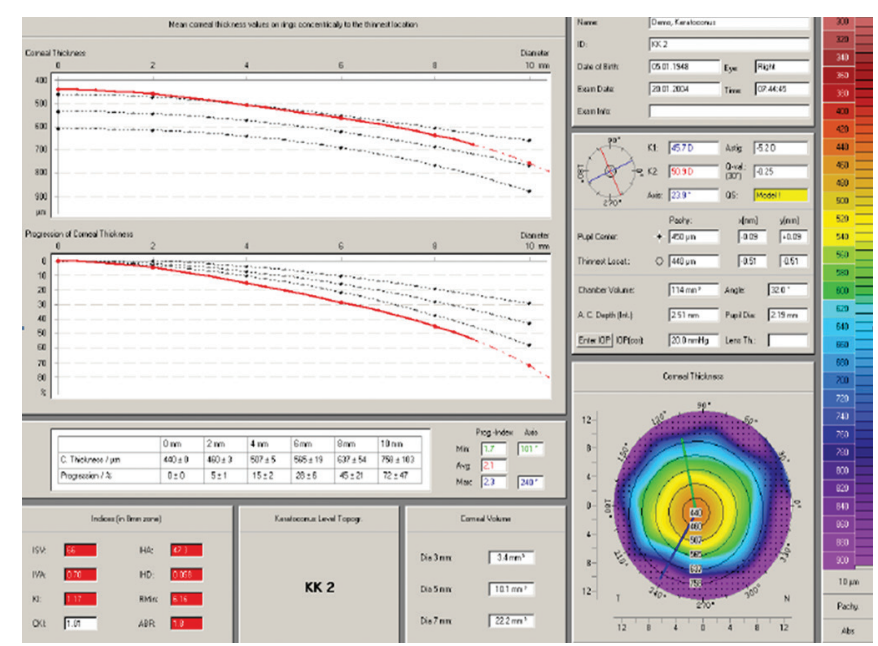

Graph 3: Anterior chamber volume in normal and moderate keratoconus. Normal subjects mean $\left( \pm 173.22 \mathrm{~mm}^{3}\right)$ and SD $\left( \pm 54.92 \mathrm{~mm}^{3}\right)$ and with significantly higher mean ACD in moderate keratoconus $\left( \pm 190.12 \mathrm{~mm}^{3}\right)$ with SD $\left( \pm 32.21 \mathrm{~mm}^{3}\right)$

in the mild, moderate, and severe keratoconus group respectively. ${ }^{9}$

In this study comparison between normal eyes and moderate keratoconus was performed and the differences in ACD and ACV values measured with Pentacam were within clinically acceptable levels in normal eyes but were not considerably clinically within accepted levels in keratoconus eyes (moderate) with significant increase in the $\mathrm{ACD}$ and $\mathrm{ACV}$ values in moderate keratoconus. According to our findings, the ACD and ACV altered significantly with progression of the disease and despite the progressive increase in the ACV with disease progression, the differences between groups were statistically significant between the moderate keratoconus group and the control group $(p=0.04)$.

Using other devices as Visante OCT and Orbascan in assessing anterior segment and comparing the results is important in order to detect accuracy and reproducibility, although results show no significant difference in ACD and ACV measurements. ${ }^{10}$

About the ACD specifically, it is a crucial parameter in the implantation of the phakic intraocular lenses (pIOLs) because they are mostly placed in the anterior chamber of the eye. This parameter should also be considered for posterior chamber intraocular lens implantation to enhance patient selection as well as biometrical estimations. ${ }^{11}$ The pIOLs are believed to be helpful in the management of the keratoconus. ${ }^{12,13}$ Hence, the investigated deepening of the ACD not only in canter but also in peripheral areas with progression of disease may propose some benefits for surgically placement of pIOLs in keratoconus patients ${ }^{14}$ as a treatment option. Identifying ACD values at paracentral area may also help in monitoring of the keratoconus ${ }^{15}$ and other surgical management of the disease. 


\section{REFERENCES}

1. Chen D, Lam AK. Reliability and repeatability of the Pentacam on corneal curvatures. Clin Exp Optom 2009 Mar;92(2):110-118.

2. Read SA, Collins MJ, Iskander DR, Davis BA. Corneal topography with Scheimpflug imaging and videokeratography: comparative study of normal eyes. J Cataract Refract Surg 2009 Jun;35(6):1072-1081.

3. Ambrósio R Jr, Caiado AL, Guerra FP, Louzada R, Roy AS, Luz A, Dupps WJ, Belin MW. Novel pachymetric parameters based on corneal tomography for diagnosing keratoconus. J Refract Surg 2011 Oct;27(10):753-758.

4. Uçakhan OO, Ozkan M, Kanpolat A. Corneal thickness measurements in normal and keratoconic eyes: Pentacam comprehensive eye scanner versus noncontact specular microscopy and ultrasound pachymetry. J Cataract Refract Surg 2006 Jun;32(6):970-977.

5. Harris WF, Malan DJ, Rubin A. The distribution of dioptric power: ellipsoids of constant probability density. Ophthalmic Physiol Opt 1991 Oct;11(4):381-384.

6. Edmonds CR, Wung SF, Pemberton B, Surrett S. Comparison of anterior chamber depth of normal and keratoconus eyes using Scheimpflug photography. Eye Contact Lens 2009 May;35(3):120-122.

7. Abolbashari F, Mohidina N, Mahdi S, Hosseini A. Anterior segment characteristics of keratoconus eyes in a sample of Asian population. Contact Lens Anterior Eye 2013 Aug;36(4):191-195.

8. Emre S, Doganay S, Yologlu S. Evaluation of anterior segment parameters in keratoconic eyes measured with the
Pentacam system. J Cataract Refract Surg 2007 Oct;33(10): 1708-1712.

9. Muftuoglu O, Ayar O, Ozulken K, Ozyol E, Akinci A. Posterior corneal elevation and back difference corneal elevation in diagnosing forme fruste keratoconus in the fellow eyes of unilateral keratoconus patients. J Cataract Refract Surg 2013 Sep;39(9):1348-1357.

10. Yazıcı AT, Pekel G, Bozkurt E, Yıldırım Y, Pekel E, Demirok A, Yilmaz OF. Measurements of anterior segment parameters using three different non-contact optical devices in keratoconus patients. Int J Ophthalmol 2013 Aug 18;6(4):521-525.

11. Hosny M, Alió JL, Claramonte P, Attia WH, Pérez-Santonja JJ. Relationship between anterior chamber depth, refractive state, corneal diameter, and axial length. J Refract Surg 2000 May-Jun;16(3):336-340.

12. Budo C, Bartels MC, Rij G. Implantation of artisan toric phakic intraocular lenses for the correction of astigmatism and spherical errors in patients with keratoconus. J Refract Surg 2005 May-Jun;21(3):218-222.

13. Leccisotti A, Fields SV. Angle-supported phakic intraocular lenses in eyes with keratoconus and myopia. J Cataract Refract Surg 2003 Aug;29(8):1530-1536.

14. Sahebjada S, Xie J, Chan E, Snibson G, Daniel M, Baird PN. Assessment of anterior segment parameters of keratoconus eyes in an Australian population. Optom Vis Sci 2014 Jul;91(7):803-809.

15. Anayol MA, Güler E, Ya c R. Comparison of central corneal thickness, thinnest corneal thickness, anterior chamber depth, and simulated keratometry using galilei, pentacam, and sirius devices. Cornea 2014 Jun;33(6):582-586. 